\title{
L'âge des amours. Genre et réciprocité érotique en Grèce archaïque
}

The age of lovemaking: gender and erotic reciprocity in Archaic Greece

\section{Sandra Boehringer et Stefano Caciagli}

\section{(2) OpenEdition}

1 Journals

\section{Édition électronique}

URL : http://journals.openedition.org/clio/12731

DOI : 10.4000/clio.12731

ISSN : 1777-5299

Éditeur

Belin

\section{Édition imprimée}

Date de publication : 1 décembre 2015

Pagination : 25-52

ISBN : 9782701194325

ISSN : 1252-7017

Référence électronique

Sandra Boehringer et Stefano Caciagli, «L'âge des amours. Genre et réciprocité érotique en Grèce archaïque », Clio. Femmes, Genre, Histoire [En ligne], 42 | 2015, mis en ligne le 01 décembre 2018, consulté le 15 mai 2020. URL : http://journals.openedition.org/clio/12731 ; DOI : https://doi.org/ 10.4000/clio.12731 


\title{
L'âge des amours. Genre et réciprocité érotique en Grèce archaïque
}

\author{
Sandra BOEHRINGER \& Stefano CACIAGLI*
}

Qui dit "âge et sexualité dans l'Antiquité » aujourd'hui pense assez spontanément à la «pédérastie grecque», pratique reconnue et valorisée à certaines périodes de l'histoire. Il faut se méfier, cependant, des évidences et des associations libres : poser la question de l'âge des partenaires dans une relation sexuelle n'est pas, dans l'Antiquité, une question qui relève des mêmes enjeux et des mêmes problématiques que ceux des sociétés occidentales contemporaines, et il serait anachronique de rechercher le lien que l'on peut établir aujourd'hui entre les questions d'âge et la sexualité dans des sociétés que l'on considère désormais comme relevant d'un régime before sexuality ${ }^{1}$.

La question de l'âge n'était pas, bien sûr, étrangère aux usages et aux normes des sociétés anciennes, et de nombreux travaux existent pour montrer la spécificité de ces catégories, nommées parfois par les chercheurs «classes d'âge $»^{2}$. Celles-ci allient des considérations de

* Les auteurs tiennent à adresser leurs remerciements à Claude Calame, Camillo Neri et Violaine Sebillotte Cuchet pour leurs suggestions et leur soutien amical et scientifique.

1 Sur cette approche de sociétés antiques, voir en particulier, l'ouvrage éponyme, cf. Halperin, Winkler \& Zeitlin (éd.) 1990, ainsi que Halperin 2000 [1990] et Winkler 2005 [1990], qui complètent l'hypothèse développée par Michel Foucault dans l'Histoire de la sexualité d'une différence radicale de dispositifs entre les sociétés antiques et les sociétés contemporaines occidentales héritières d'une scientia sexualis. Pour un état des lieux des récents travaux sur les questions du genre et de la sexualité en Grèce ancienne, voir Boehringer 2012, Hubbard (éd.) 2014a, Blondell \& Ormand (éd.) 2015.

2 Cf. Jeanmaire 1939, Brelich 1969 et Calame 1977. Pour une analyse épistémologique du recours à ces catégories d'analyse, voir Calame 2003. 
genre, de statuts et de spécificités géographiques et culturelles (pour la Grèce par exemple, les usages différaient sensiblement d'une cité à l'autre) ${ }^{3}$. L'intérêt que nous portons, chercheurs occidentaux du XXI ${ }^{\mathrm{e}}$ siècle, au lien qui existe entre l'âge et la sexualité trouve son origine dans des préoccupations actuelles et se formule en des termes contemporains. C'est cette formulation qu'il convient d'expliciter, afin de pouvoir percevoir, dans un second temps, la grande différence qui nous sépare du régime de l'érotisme de ces sociétés antiques.

Cette étude se propose de définir préalablement les termes actuels de notre enquête, puis de donner un panorama des acquis des récents travaux sur l'Antiquité autour des questions d'âge et de sexualité, souvent interprétées en termes de relation symétrique ou asymétrique. Enfin, ce point sera développé par une analyse fondée sur une étude de la poésie grecque de l'époque archaïque ${ }^{4}$ qui permettra de déconstruire l'idée préconçue que les relations homoérotiques entre femmes seraient plus égalitaires que la relation homosexuelle masculine et de reprendre à nouveaux frais la question de la réciprocité érotique.

\section{Des sociétés before sexuality}

Les Anciens, comme l'ont montré de nombreux chercheurs, pour la plupart à la suite des travaux de Michel Foucault, ne se définissaient pas personnellement en fonction d'une «sexualité», et le sexe de la personne désirée n'était pas un critère pour définir et catégoriser un individu. Les Anciens n'ont jamais considéré que pouvaient être regroupées dans une même catégorie d'individus des personnes, hommes et femmes, de tous statuts (citoyens, métèques, étrangers, esclaves), de toutes origines et de tous milieux sur le simple critère qu'ils étaient attirés par des personnes de l'autre sexe, ou sur le critère d'une attirance pour des personnes du même sexe. L'étude des

3 Pour une réflexion sur la façon dont les études des XIX et $\mathrm{XX}^{\mathrm{e}}$ siècles portant sur les classes d'âge ont croisé les questions de sexualité et d'homosexualité, en particulier dans le cas de populations doriennes, voir Boehringer 2014.

4 Cette étape de la réflexion s'attachera plus spécifiquement à une étude de la poésie grecque non épique de l'époque archaïque (VII--VI ${ }^{e}$ siècle avant J.-C.). Sans précision de notre part, les siècles mentionnés dans cet article s'entendent avant J.-C. 
documents montre clairement que la première distinction perçue par les Anciens n'est pas celle du sexe mais celle qui oppose les individus libres à ceux qui ne le sont pas, ceux qui disposent de leur corps et ceux dont le corps appartient à un maittre - à savoir une très importante proportion de la population, la population servile 5 . De façon générale, quand les Grecs disent ándres ou gynaîkes, ils désignent uniquement la population citoyenne, ou au mieux les individus libres (citoyens, affranchis, métèques, étrangers): de ce fait ces termes n'ont pas le même signifié qu'ont, aujourd'hui, les mots « hommes » et « femmes ».

Ajoutons que, dans la mesure où aucune répression ne vient discriminer les individus attirés par des personnes du même sexe qu'eux, il n'y a pas de catégories de personnes dont l'expérience commune serait au fondement d'une culture spécifique. L'étude de Michel Foucault et, quelques années plus tard, celle de David Halperin, sur les discours qui développent une comparaison entre l'amour des garçons et l'amour pour les femmes (dans l'Anthologie grecque, chez Lucien, Plutarque et Achille Tatius) ${ }^{6}$, ont montré que ces oppositions rhétoriques et ponctuelles non seulement excluaient le point de vue des femmes et la possibilité des relations entre hommes adultes (qu'aucun des personnages ne prend à son compte), mais surtout exprimaient des question de goût n'engageant pas toute une vie et encore moins une identité psychologique. Si l'on ajoute à cette réflexion le fait que les relations entre femmes ne sont jamais inclues dans ces possibilités de la comparaison ${ }^{7}$, le constat est simple: pas plus qu'il n'existe d'identité «femmes » ou «hommes » (puisque le statut est le premier élément discriminant qui empêche toute conscience d'une «condition féminine ${ }^{8}$ ), l'hétérosexualité et l'homosexualité, en tant que telles,

5 Sur la vaste question de la population servile en Grèce antique, voir, entre autres, les nombreux travaux de Finley et ceux de Garlan 1982 ; pour l'importance des esclaves dans le domaine des « services sexuels », voir l'étude récente de Cohen E. 2014.

6 Pour une étude de la comparaison érotique (du point de vue du citoyen grec), voir Foucault 1984 : 243-261 et Halperin 1992.

7 Pour une étude de la comparaison érotique incluant la question des femmes, voir Boehringer 2007b.

8 Il ne s'agit pas de dire qu'une «histoire des femmes» serait inutile, il s'agit simplement de souligner que «les femmes» ne constituent pas une catégorie sociale homogène d'un point de vue émique. 
n'existent pas dans l'Antiquité. En Grèce ancienne, le caractère désirable d'un corps n'est pas lié à son sexe ${ }^{9}$ : cependant, dans ces questions de goûts (et non d'identité), l'âge peut intervenir comme un facteur explicite.

\section{Le mariage grec : une asymétrie complète}

Solon, poète et législateur athénien $\mathrm{du} \mathrm{VI}^{\mathrm{e}}$ siècle, apporte des indications sur les perceptions grecques des âges de la vie d'un homme libre ${ }^{10}$. Malgré la subdivision arbitraire de la vie humaine en périodes de sept ans, on y peut distinguer cinq moments : l'enfance, quand le paîs n'est pas pubère; l'adolescence, caractérisée par une condition éphémère, car la «fleur» de la peau change et les joues se couvrent de poils ${ }^{11}$; la jeunesse adulte, quand l'homme de 21 ans devient néos et n'est plus népios ${ }^{12}$, car il est au sommet de sa force et vertu ; la maturité, quand l'homme (anér) est formé, qu'il se marie autour des 30 ans et qu'il a acquis la prudence ; enfin, la vieillesse, quand la personne a perdu sa force, mais se trouve au sommet de la sagesse ${ }^{13}$.

Cette subdivision ne s'adapte pas aux femmes ${ }^{14}$ : chez elles, en effet, on trouve l'enfance; la nubilité (parthenía), qui dure plus ou

9 Sur le caractère érotique du corps en Grèce ancienne, voir la récente étude de Stansbury-O’Donnell 2014, qui propose également une synthèse de l'historiographie sur cette question.

10 Les poèmes de Sappho et Alcée sont cités selon l'édition de Voigt [= V., 1971] ; ceux d'Alcman selon l'édition de Davies [= D., 1991] ; ceux de Mimnerme et de Théognis selon la deuxième édition de West [= W. $\left..^{2}, 1989-1992\right]$; ceux d'Anacréon selon l'édition de Page [= P., 1962].

11 La fleur, ánthos, a une valeur érotique, cf. infra.

12 Dans l'Odyssée, II, 312-315, Télémaque revendique, face aux prétendants, le fait qu'il n'est plus un népios. Sachant qu'Ulysse a combattu durant dix années et que son retour a duré dix ans, et étant donné que Télémaque était un petit enfant quand son père est parti faire la guerre (cf. Eustathe, Commentaire à POdyssée, 1956, 20 sq.), on peut en déduire qu'il a environ vingt ans.

13 Solon, fr. 27 [W.2] : l'enfance (v. 1 sq.) ; l'adolescence (v. 3-6); la jeunesse adulte (v. 7 sq.) ; la maturité (v. 9-14) ; la vieillesse (v. 15-18).

14 Cf., par exemple, Scholies au v. 356 de l'Alexandre de Lycophron : voir aussi Calame 1996 : 130-145. 
moins quatre ans ${ }^{15}$ et qui est caractérisée par le désir érotique que la jeune fille suscite ${ }^{16}$; la condition - en principe, très brève - de jeune épouse (nýmphê), qui n'a pas encore enfanté ; celle de femme adulte (gyné), qui est désormais devenu mère ; la vieillesse, quand la femme a perdu la possibilité d'enfanter ${ }^{17}$. De façon générale, et malgré les différences chronologiques et spatiales, cette asymétrie entre les classes d'âges des hommes et celles des femmes se retrouve à l'âge du mariage $^{18}$ : les premiers se marient autour des 30 ans $^{19}$, alors que les femmes sont données à marier autour de 18 ans; ainsi, les hommes étaient jeunes adultes (néol) pour une dizaine d'années, tandis que les femmes l'étaient généralement pour une année seulement (nýmphai).

La différence de parcours de vie entre les hommes et les femmes libres apparaît d'emblée dans ces subdivisions. La future épouse, ainsi, est "donnée en mariage » par son père ou son tuteur, parfois promise très tôt et avant même que la cérémonie du mariage (le gámos) ne soit célébrée. Il n’y a pas d'âge minimum légal qui ferait autorité de façon homogène, mais les documents permettent de donner des tendances et des coutumes ${ }^{20}$. Au VIII ${ }^{\text {e }}$ siècle, Hésiode évoque l'âge de 18 ans environ ${ }^{21}$; le code de Gortyne, en Crète, au Ve siècle, fixe l'âge de 12 ans au plus tôt ${ }^{22}$; au IVe siècle, Aristote parle

15 Cf. Hésiode, Les Travaux et les jours, v. 695-705.

16 Cf., par exemple, Pindare, Pythiques, 3, 8-23 et Sappho, fr. 112 [V.]

17 Dans Aristophane, Lysistrata, v. 641-645, les femmes du chœur semblent faire allusion à des classes d'âges : arréphores, aletrídes, ourses et canéphores. Pourtant, ces fonctions religieuses ne concernaient pas toutes les Athéniennes, ce qui pourrait indiquer qu'elles n'ont pas de lien direct avec les classes d'âges (cf. Caciagli 2011 : 308 sq.). Sur les questions philologiques qui concernent ces vers et leur interprétation, cf. Perusino 2002.

18 Sur le mariage en Grèce ancienne, voir plus particulièrement les études de : Leduc 1991, Oakley \& Sinos 1994 et Glazebrook \& Olson 2014.

19 Cf. Hésiode, Les Travaux et les jours, v. 696.

20 En Grèce antique, l'âge des individus est indiqué par l'année en cours et non le nombre des années révolues. Par exemple, la quatorzième année désigne l'année des treize ans (soit un âge entre treize et quatorze ans).

21 Hésiode, Les Travaux et les jours, v. 695-705: le poète conseille qu'une fille soit donnée en mariage quatre ans après les premières règles (qui apparaissent autour de 13/14 ans). Sur ce point, cf. Brulé 1987 : 361 sq.

22 Code de Gortyne, Inscriptiones Creticae, IV, 72, col. XII, 1. 18. 
de 14 ans comme étant l'âge minimal, à Athènes, pour marier les filles épiclères ${ }^{23}$. Enfin, les jeunes filles pouvaient être mariées avant d'avoir leurs premières menstruations ${ }^{24}$. Si la différence d'âge habituelle était de dix ou quinze années, les jeunes filles pouvaient avoir des époux bien plus âgés encore. Les usages, quelles que soient l'époque et la région, instituent ainsi une asymétrie d'âge dans la relation conjugale des individus libres.

Une question se pose alors : cette asymétrie dans l'âge du mariage fait-elle des hommes mariés des individus aux penchants pédophiles? Tout le monde percevra l'anachronisme de cette question. Pourtant son équivalent pour les relations sexuelles entre hommes a souvent circulé, preuve que les regards vers le passé sont toujours informés de codes moraux du présent.

\section{Jeunesse et consentement aux relations érotiques}

Aujourd'hui, en droit français, où la majorité sexuelle est de 15 ans, la sexualité des mineurs est soit interdite (entre une personne de plus de quinze ans et les personnes de moins de 15 ans), soit encadrée (pour les personnes entre 15 et 18 ans) ${ }^{25}$ : cette législation favorise, en creux, les relations entre personnes du même âge. En Grèce, la différence d'âge est, au contraire, un élément qui rend la relation entre les amants estimable et digne de respect: elle s'affiche dans les lieux publics comme dans les banquets, elle est parfois même codifiée par la cité26, les mythes racontent à l'envi des histoires d'amour et d'enlèvements érotiques entre un dieu puissant et un jeune mortel ${ }^{27}$.

23 Il s'agit des filles qui, à la mort de leur père, sont les seules descendantes : elles sont alors mariées très tôt à un proche parent pour que le patrimoine foncier, le klêros, reste dans la famille. Cf. Aristote, Constitution des Athéniens, 56, 7. Cf. aussi Xénophon, Économique, 7, 5.

24 Cf. Brulé 1987 : 366.

25 Cf. les articles 227-25 et 222-27 du Code pénal (France).

26 Sur certaines formes de l'homoérotisme masculin et les contextes de sa valorisation, voir, entre autres, Dover 1982 [1978], Halperin 2000 [1990], Lear 2014.

27 Voir par exemple Pindare, Olympique, 1, qui évoque l'enlèvement du jeune Pélops par le dieu Poséidon. Pour une étude des mythes dits d'homosexualité masculine, voir Sergent 1996 [1984]. 
Le critère de "personne ayant autorité » qui fonctionne aujourd'hui comme un interdit dans le code pénal français pour la sexualité des mineurs est, à l'inverse également, un élément qui favorise, dans les représentations communes grecques, l'aspect éthique de cette relation. La différence d'âge peut être chantée dans le cadre du banquet aristocratique à l'époque archaïque ${ }^{28}$ et c'est un aspect qui est souligné par Platon dans ses dialogues : la «bonne pédérastie » est celle qui mène vers la philosophie ${ }^{29}$.

Cependant, contrairement à ce que certains commentateurs modernes ont voulu voir dans leur recherche d'explication à ces pratiques exotiques ${ }^{30}$, cette dimension d'autorité avec possibilité de transfert de savoir via une relation pédagogique, même si elle peut être très importante, n'est pas un élément nécessaire à l'existence sociale de cette relation érotique adulte/jeune homme de façon générale. De nombreux documents attestent d'une attirance érotique envers les corps de jeunes garçons sans qu'elle ne soit justifiée par un élan intellectuel ou moral, et sans qu'elle ne devienne objet d'un interdit d'ordre légal ou de condamnation morale ${ }^{31}$.

L'âge du jeune homme n'est pas un critère qui le constituerait comme un partenaire sexuel interdit, car vulnérable ; au contraire, sa jeunesse en fait un partenaire privilégié. En Crète, selon un récit de l'historien Éphore (IVe siècle avant J.-C.) rapporté par Strabon ${ }^{32}$,

\footnotetext{
28 Cf. infra.

29 Voir Brisson 2006 ; Renaut 2015 et Renaut 2016.

30 Pour une mise au point théorique sur les tentations étiologiques, voir le commentaire de David Halperin sur l'œuvre de Harald Patzer : «En purifiant la pédérastie du désir sexuel et en y voyant non pas l'expression d'une préférence personnelle mais au contraire une forme de rituel social (la reléguant ainsi dans une catégorie d'activités séparées de la vie quotidienne normale et seulement accomplie dans des circonstances spécialement prescrites), H. Patzer continue en réalité d'assigner le lieu ordinaire de l'érotisme à l'activité hétérosexuelle - même pour les Grecs malgré leurs diverses singularités sexuelles - et, par conséquent, celle-ci reste le mode privilégié et normal de la sexualité humaine. » (Halperin 2000 [1990] : 83-104).

31 Voir par exemple les documents réunis par Dover 1982 [1978] ou les images commentées par Lear in Cantarella \& Lear 2008, où rien ne vient souligner la nécessité d'un échange intellectuel ou « initiatique ».

32 Strabon, Géographie, X, 4, 21, qui cite Éphore (FgrHist, 70, F 149).
} 
l'enfant (ho paîs) enlevé par son amant (ho erastếs) avec l'autorisation de tous reçoit éloges et cadeaux, une fois revenu dans la cité, et rend compte du fait qu'il n'a pas subi de violence physique (bía). C'est bien avec son consentement - et avec l'accord de la communauté - que la relation érotique s'est produite. Dans les représentations iconographiques ou dans les poèmes érotiques se trouvent mis en scène des garçons qui refusent (provisoirement ou définitivement) les avances de leur séducteur ${ }^{33}$. Le consentement du paîs à la relation est recevable et son amant n'est de ce fait pas un violeur ni un pervers mû par des désirs inavouables. Si, par ailleurs, cette personne joue un rôle dans la formation du jeune homme et s'il est de noble famille, et de bonne réputation, la relation n'en est que plus valorisée. Ainsi, dans le Banquet de Xénophon, le riche Callias, amoureux du jeune Autolycos, invite son aimé et son père à la soirée qu'il organise dans sa maison du Pirée ${ }^{34}$, qui accepte bien volontiers. Nous voilà loin des cadres contemporains de la sexualité surveillée des mineurs.

\section{Érōs : un élan et non une caractéristique identitaire}

Pour les Anciens, être frappé par érōs signifiait être mû par un élan immaitrisable, être envahi par une force extérieure ${ }^{35}$. La personne frappée par érōs est parfois désignée par le terme d'erastés ou d'erôn (participe présent actif): leur équivalent féminin sous une forme substantivée est très rare ${ }^{36}$. La personne qui est la cause de cet élan amoureux est désignée par le terme d'erómenos/eroménè. Pour autant, ces termes ne désignent pas une identité. Formé sur le radical verbal d'erân, il s'agit pour le premier d'un nom d'agent et, pour le second, d'un participe présent substantivé - la forme est passive (-menos). Cependant,

33 Voir ainsi les jeux et les attitudes dans les scènes de cour dans les représentations iconographiques (Lear in Cantarella \& Lear 2008 : 38-62).

34 Xénophon, Le Banquet, 1, 2-4.

35 Sur érōs et ses effets, voir Calame 1996.

36 Il existe des attestations d'un usage non substantivé du participe au féminin du verbe erân ainsi que l'emploi, dans la comédie, du terme andrerástria. Le terme de gynaikerástria apparaît dans un papyrus de la fin du II siècle-début du III de notre ère, pour qualifier Sappho (P. Oxy. 1800, fr. 1). Ce point nécessiterait une étude développée que nous n’avons pas l'occasion de mener ici. 
le sens même du verbe erân ("être frappé par érōs») et sa construction avec un génitif (et non un complément d'objet direct) ${ }^{37}$ ne permet pas de voir dans ce passif grammatical un sens passif fondé sur une répartition des rôles érotiques entendu en terme de dominant/dominé ou encore de pénétrant/pénétré ${ }^{38}$. Les deux termes désignent simplement les deux amants, de façon asymétrique et contextuelle.

Dans le vocabulaire utilisé dans des contextes évoquant des relations amoureuses entre hommes, l'objet de l'amour éprouvé n'est pas indiqué par la morphologie du terme utilisé comme c'est le cas des désignations contemporaines homo/hétérosexualité ou pédérastie. Le verbe paidophileîn ou ses dérivés apparaissent à trois reprises dans le contexte de la poésie sympotique (poésie chantée lors des banquets) ${ }^{39}$, mais sans désigner une catégorie de personnes. De même, les termes de paiderastía, paiderasteîn et paiderastés sont peu utilisés pour la période du VIII au II e siècle avant J.-C. : la quasitotalité des usages de ce terme apparaît à l'époque romaine, chez les Pères de l'Église, les auteurs grecs d'époque romaine impériale (Lucien, Plutarque, Athénée), les auteurs techniques (magie, astrologie, médecine). Et sur la douzaine d'occurrences attestées pour

37 Cf. Apollonius Dyscole, Syntaxe, III 172.

38 Selon Dover, le suffixe actif désigne la pratique érotique « active », interprétée en terme de pénétration phallique et cet acte exprime un rapport hiérarchique (statutaire ou symbolique): les codes de représentation dans l'iconographie (l'évitement d'une « humiliation » du jeune homme par une pénétration anale et la préférence pour une représentation des relations érotiques sous la forme de pénétration intercrurale) viendraient confirmer cette lecture. C'est en grande partie les analyses de Dover qui ont mené Michel Foucault à développer une interprétation des relations érotiques en terme d'isomorphie ou d'hétéromorphie, et à analyser le "problème des garçons » pour les Grecs, celui de domination sexuelle d'un jeune individu libre. L'interprétation des relations érotiques sur le modèle actif/passif, pénétrant/pénétré et dominant/dominé (voire masculin/féminin ou efféminé) est également développée par David Halperin et John Winkler à la fin des années 1980 : là, les critères d'âge sont primordiaux. De nombreux chercheurs, aujourd'hui, nuancent ces interprétations tout en restant attachée à la base théorique fondamentale de cette lecture, celle d'une société qui ne connait pas le dispositif actuel d'une sexualité identitaire. Pour une synthèse des nombreux débats sur cette question, voir Ormand 2014. Théognis, v. 1345 et 1358 [W.2] ; Solon, fr. 25 [W.2]. 
la période classique, sept occurrences apparaissent chez Platon (six dans le Banquet) ${ }^{40}$, une chez Xénophon (pour caractériser un soldat d'Olynthe qui combat mieux entouré de beaux garçons ${ }^{41}$ et les rares autres apparaissent de façon isolée : un hapax (c'est-à-dire un mot qui n'apparaît qu'une seule fois dans tout le corpus) chez l'orateur Isée (le verbe katapaiderasteîn) ${ }^{42}$, une insulte chez Aristophane (une seule occurrence, placée sur le même plan que « espèce de coucheur !») ${ }^{43}$ et dans quelques fragments, essentiellement comiques (titres de pièces dont le texte est perdu ou un vers isolé) ${ }^{44}$.

De plus, il y a une explication à l'emploi répété (en comparaison avec le peu d'occurrences dans le reste du corpus de la même période) du terme paiderastía chez Platon : il s'agit, pour le philosophe, de prouver qu'une certaine forme d'amour permet la pratique de la philosophie ${ }^{45}$. Pourtant, alors même que l'on pourrait considérer que ce texte consacre la pédérastie comme institution et comme vocable, la relation entre le jeune et bel Alcibiade et Socrate, qui se refuse à ses avances, subvertit les usages traditionnels ${ }^{46}$.

À l'époque archaïque et classique, ce que nous appelons "pédérastie grecque» ne constituait pas une catégorie sexuelle particulière séparée des autres élans érotiques. Sappho comme Alcée ou les Theognidea parlent d'érōs. Lorsqu'il évoque la valeur pédagogique accordée à l'amour des garçons à Sparte du temps de son législateur

40 Platon, Symposium, 181c, 184c, 192b (3 fois), 211b ; Phèdre, 211b.

41 Xénophon, Anabase, VII, 4.7-11.

42 Isée, Sur l'héritage d'Aristarque, 25,2: d'après ce que le contexte permet de déduire, ce verbe désigne le fait de ruiner son ô̂kos en raison des dépenses occasionnées par la fréquentation des paîdes.

43 Aristophane, Acharniens, v. 265.

44 Antiphane, Paiderastés, fr. 179 [K.-A.] ; Diphile, Paiderastaí, fr. 57 [K.-A.] ; et Eubule, fr. 130 [K.-A.]

45 «L'unité du Banquet se fait dès lors autour d'un Éros qui prend les traits de cette séduction qui se manifeste dans l'éducation, séduction sexuelle dans le cas de la paiderastía, séduction dialogique dans le cas de la philosophia », analyse Luc Brisson (2015).

46 Sur Platon et plus particulièrement sur le discours de Pausanias dans le Banquet, voir l'étude éclairante de Renaut 2015. 
Lycurgue, Xénophon utilise simplement le terme d'erastếs ${ }^{47}$, et chez Aristote, comme nous l'avons vu, et dans les très nombreux contextes évoquant des relations érotiques ${ }^{48}$, les amants, frappés par érōs, même s'ils aiment des garçons, sont des erôntes et non des paiderôntes. Ainsi, si des pratiques que nous définissons comme pédérastiques apparaissent en nombre dans les documents qui nous sont parvenus, force est de constater que les Grecs ne «se disaient» pas pédérastes, comme l'on peut parler de «se dire lesbienne $»^{49}$ aujourd'hui. Les personnes engagées dans de telles relations occupaient des rôles ou des fonctions dans des contextes particuliers, mais ils n'avaient pas le sentiment d'avoir une identité particulière (d'éraste ou d'éromène), ni l'envie de construire une culture spécifique, qu'elle soit marginale ou majoritaire. En Grèce archaïque et classique, il n'y avait pas d'affirmation identitaire à aimer les jeunes gens, de ce fait, ni de fierté ni de honte ${ }^{50}$.

Bien évidemment ces relations érotiques ont donné matière à une intense réflexion et à une riche création poétique et artistique dans les sociétés grecques, mais il ne s'agit en rien d'une production spécifique ou isolée: les vases dit "pédérastiques» circulaient dans des contextes où des joueuses de flûtes pouvaient éveiller les sens des mêmes convives ${ }^{51}$, et où diverses formes de couples (hommes et femmes, hommes ensemble, satyres entre eux ou avec ménades) apparaissaient sur les coupes qui circulaient, contribuant au luxe de la réception. La catégorie "pédérastique » est, pour les périodes qui nous occupent, une catégorie anachronique, construite par des

47 Xénophon, Constitution des Lacédémoniens, 2.12-13.

48 Voir les nombreux textes évoquant des relations érotiques dans les recueils et anthologies : Hubbard 2003 et Boehringer \& Tin 2010.

49 Nous reprenons ici le titre d'une étude récente, Se dire lesbienne (Payot, 2010) de Natacha Chetcuti, où l'importance d'une affirmation de soi en ces termes illustre le poids actuel de la sexualité dans les identités. Ajoutons qu'en Grèce ancienne, le verbe lesbiázein renvoyait à des pratiques sexuelles avec des hommes, dont la fellation. Sur le lien complexe entre Sappho, Lesbos et l'homosexualité féminine dans l'histoire ancienne, voir Boehringer 2007a : 61-66 et 211-214 et Gilhully 2015).

50 Si honte il y avait, c'était en cas d'excès ou de dépense financière, et non en raison d'une anormalité psychologique ou d'une déviance.

51 Voir l'étude de Cantarella \& Lear 2008 et une mise au point dans Lear 2014. 
chercheurs influencés par le discours moral des auteurs grecs postérieurs et pris dans le discours classificateur d'une scientia sexualis.

\section{Des aimé(e)s, des amants : l'asymétrie érotique dans la poésie archaïque}

S’il n'existe pas de catégorie stricte désignée par «pédérastie » et s'il n'y a pas d'identité liée à une (anachronique) "orientation sexuelle», c'est en terme d'asymétrie qu'il est fructueux d'interpréter les relations érotiques apparaissant dans la poésie grecque non épique de l'époque archaïque : la réalité des pratiques était en effet bien plus variée que certaines schématisations contemporaines le font croire.

Avant de continuer, une précision d'ordre formel est nécessaire. La poésie archaïque est radicalement différente de la poésie moderne : étant donnés son caractère oral et le rapport direct du poète avec son auditoire, on la considère comme une poésie pragmatique, dans le sens où elle a un lien direct avec la réalité, ou du moins avec celle de sa première performance. Sans pour autant être un simple reflet de la réalité, les poèmes de l'époque archaïque peuvent être considérés comme une source importante, sinon fondamentale, pour connaitre les pratiques sociales de leur époque ${ }^{52}$. Ces poèmes sont issus généralement du contexte du banquet aristocratique ${ }^{53}$, qui était idéologiquement central en Grèce archaïque et classique, car il était le moment où les hommes discutaient, entres autres, des questions politiques et sociales; ce moment, comme on le verra, était décisif aussi pour l'éducation des jeunes aristocrates.

Dans la poésie grecque, la différence d'âge caractérise généralement les relations des hommes autant avec d'autres hommes qu'avec des femmes libres : la jeune mariée et l'aimé ont environ le même âge, et l'époux de la première et l'amant du second sont plus âgés que leur partenaire. Cette forme d'asymétrie a des retombées dans deux domaines décisifs pour la société grecque: le banquet

52 Cf. Gentili 2006 [1984] : 15-47; Rösler 1984; Calame 1998; Calame 2005 : 30 sq. ; Caciagli $2011: 11-19$.

53 Cf. Rösler 1980 : 26-41 et Vetta 1983 : XIII sq. Sur le banquet grec, cf. Murray 1990 (éd.) et Schmitt-Pantel 1992. 
aristocratique, où les adolescents issus des nobles familles étaient instruits par le fait d'écouter les discussions des adultes et de voir leurs gestes, en assimilant ainsi les coutumes propres à leur classe sociale; la cérémonie de mariage, le gámos, où la jeune fille libre devenait une femme adulte et suscitait un désir légitime et reconnu. La norme que nous venons d'esquisser connaissait, certes, des exceptions : une absence d'asymétrie d'âge dans le mariage ${ }^{54}$ ou des rapports symétriques entre hommes ${ }^{55}$. La poésie grecque archaïque, cependant, pour autant que les sources nous permettent de le savoir, ne parle pas de ces derniers : ceci ne signifie pas, bien évidemment, que ce type de relations n'existait pas dans la Grèce archaïque, mais qu'il n'avait pas d'intérêt social pour les groupes qui se réunissaient dans les banquets aristocratiques. Le seul exemple que l'on pourrait évoquer à ce propos sont les vers 1062 sq. des Theognidea ${ }^{56}$ : «On peut, quand on est jeune, dormir toute la nuit près d'une personne du même âge (homêlix) / et satisfaire ses désirs amoureux $»^{57}$. Ce passage, toutefois, est problématique, en particulier car le terme grec d'homêlix peut avoir comme référent autant un homme qu'une femme. Puisqu'on ne connait pas les circonstances qui furent le contexte de la première performance de ces vers, homêlix peut, certes, renvoyer à un rapport entre deux hommes du même âge. Toutefois, il peut renvoyer

54 Des jeunes gens (cf. Odyssée, XVI, 245 sq., XXII, 30, XIV, 131) sont les prétendants de Pénélope, qui, elle, doit avoir au moins quarante ans (puisqu'elle a un fils de plus de vingt ans).

55 Sur l'érōs masculin symétrique, cf. Hubbard 2003: 5 et 2014b: 128-130. Les exemples qu'il offre pour étayer son argument restent à l'état d'hypothèse. Outre Théognis, v. 1362 sq. [W.2] (commenté ci-dessus), les autres extraits relevés par Hubbard sont : Pindare, Pythiques, 10, 57-60 (Hippoclès est objet d'admiration, sans valeur érotique); Théognis, v. 1319-1322 [W.2] (les statuts de paîs et de néos sont différents, cf. infra) ; Platon, Phèdre, 240c (le plaisir entre jeunes du même âge pourrait ne pas être érotique). Les vases sont difficiles à interpréter, car leurs représentations ne sont pas réalistes (voir Lear 2014 : 107).

56 Le corpus des Theognidea est une sorte d'anthologie d'élégies de plusieurs auteurs, généralement d'époque archaïque: ce corpus a été transmis sous le nom de Théognis, en raison du sceau des vers 22 et suivant (cf. West 1974 : 40-46 ; Vetta 1980 : XI-XXVII ; Colesanti 2011 : 1-33). Ce sceau, pourtant, se réfère uniquement à l'élégie des vers 14-25 ou, à la limite, à une section du corpus.

57 Théognis, v. 1062-1063 [W.2]. 
aussi à un féminin, ce qui n'implique pas forcément un rapport égalitaire: il peut s'agir, par exemple, d'une relation avec une courtisane du même âge. En outre, homêlix pourrait faire référence au fait que les deux partenaires sont, tout simplement, jeunes : il faudrait alors interpréter «du même âge » avec un sens moins limité 58 .

Le moment idéal pour érōs, en effet, est avant tout celui de la jeunesse, et c'est ce que le poète Mimnerme rappelle dans ces vers : «Le rapport réciproque (philótês) ${ }^{59}$, les dons doux comme le miel et le lit... sont les fleurs désirées de la jeunesse (hébè) pour les hommes (andrásin) et pour les femmes (gynaixín) $»^{60}$. Ce passage trouve un parallèle dans un passage des Theognidea. Là, le poète invoque Cypris de le libérer des soucis amoureux et de lui donner la sagesse, liée à la fin de la jeunesse (hếbê) ${ }^{61}$. Apparaît, ici, le fait que l'âge sensible à l'érōs est celui de la jeunesse. L'amant se rapporte ainsi au jeune adulte, ou néos ${ }^{62}$. Que reste-t-il, alors, de l'asymétrie généralement considérée comme normative dans les relations homérotiques entre un adolescent et un homme adulte?

Dans les vers des Theognidea, l'aimé et l'amant sont souvent chantés et mis en scène comme ayant des rôles établis et non paritaires. En ce qui concerne l'aimé, il est souvent représenté comme s'il n'était pas dominé par les œuvres d'Aphrodite, bien qu'il ait ses dons. Les vers 1299-1304 montrent le paîs accusé de fuir l'amant, qui est contraint de le poursuivre ${ }^{63}$. Pour convaincre le paîs de s'arrêter, on lui rappelle alors que le don de Cypris est de courte durée : la fleur (ánthos) de l'adolescence est rapide à se faner ${ }^{64}$ et le paîs doit penser au

58 Cf. Van Groningen 1966 : 394

59 Philótês est souvent traduit par «amour » ou « amitié » : l'aspect fondamental de sa signification est celle d'un pacte qui implique réciprocité entre les contractants (cf. Benveniste 1969 : I, 335-353).

60 Mimnerme, fr. 1, 3-5 [W.2].

61 Théognis, v. 1323-1326 [W.2].

62 Cf. supra. Selon Plutarque, Lycurgue, 17, 1, les amants de Sparte étaient des néoi de bonne réputation. Sur le rapport problématique entre l'idée de la Sparte archaïque et la réalité de l'époque impériale, cf. Kennell 1995 : 83 sq., 87-97, 143-148.

63 Cf. infra Sappho, fr. 1, 21 [V.].

64 Cf. Théognis, v. 1323-1326 [W.2]. 
moment où, lui aussi, devra subir les tourments et les affres qu'imposent les œuvres d'Aphrodite-Cypris.

Dans ce contexte, c'est la barbe qui marque la fin de la condition de l'aimé ${ }^{65}$ : aux vers 1327 et suivant, un poète des Theognidea assure qu'il louera le paîs jusqu'au moment où sa joue restera lisse. L'absence de barbe est un élément qui permet un rapprochement entre le paîs et la parthénos, les deux objets du désir des néoi: dans les vers 1283-1294 des Theognidea, par exemple, le paîs qui fuit l'amant est comparé au paradigme mythique d'Atalante, une parthénos en âge de se marier, qui fuit ses prétendants ${ }^{66}$. Anacréon, poète du $\mathrm{VI}^{\mathrm{e}}$ siècle actif principalement à Samos et Athènes, dit, quant à lui, que le garçon aimé a un regard de jeune fille ${ }^{67}$. Dans le domaine érotique, donc, l'absence de poils et, notamment, de la barbe semble produire une forme d'identification entre la jeune fille et le jeune garçon.

La condition transitoire de l'aimé est liée au rôle qu'il joue dans la relation avec son amant plus âgé. Si celui-ci est dominé par le désir que le paîs suscite ${ }^{68}$ et demande la cháris (au sens de faveur, dans une relation d'échange), c'est au paîs de donner ${ }^{69}$. Les vers 1263-1266 esquissent bien la réciprocité de la relation pédérastique: malgré l'asymétrie du rapport, la cháris exige que le paîs donne une réponse en retour des biens reçus. Ces biens, ce sont les cadeaux que les amants faisaient aux aimés, mais aussi les bons conseils que le jeune adulte donnait à l'adolescent (d'où la valeur éducative de cette relation érotique). Pourtant, la poésie sympotique souvent chante le fait que le paîs ne répond pas aux requêtes de l'amant.

Jeune garçon (paîs), au regard de jeune fille (parthénos),

je te cherche, mais tu ne m'entends pas ;

tu ne sais pas que tu tiens

les rênes de mon âme ${ }^{70}$.

\footnotetext{
65 Cf. supra Solon, fr. 27 [W.2].

66 Sur ces vers et pour une analyse en terme de genre, voir, entre autres, Boehringer \& Chabod, à paraître.

67 Cf. Anacréon, fr. 360 [P.] (infra).

68 Cf. Théognis, v. 1235-1238, 1344, 1350, 1388 sq. [W.2].

69 Cf. Théognis, v. 1329 sq. [W.²].

70 Anacréon, fr. 360 [P.] (trad. Caciagli).
} 
Plusieurs éléments d'une asymétrie érotique sont nettement visibles ici : l'amant, dominé par le désir amoureux, cherche l'aimé qui ne répond pas à ses requêtes. Ce passage peut se comprendre également comme faisant référence au moment d'une attente souhaitable: le paîs ne doit pas tout de suite donner son accord à l'érōs, même si la réciprocité érotique est le but de la relation entre amant et aimé. Les vers 1319-1322 des Theognidea, où la question de la « persuasion » de l'aimé apparaît, offre une autre illustration des rôles de l'amant et l'aimé.

Jeune garçon (paîs), puisque la déesse Cypris t'a accordé cette faveur (cháris) charmante, et que tous les jeunes gens (néor) ne pensent qu'à ta beauté (eîdos). prête oreille à mes paroles et, pour me complaire (cháris), fais-les pénétrer dans ton cœur.

Tu sais que le désir amoureux (érōs) est pour un homme (anér) un fardeau pénible à porter. ${ }^{71}$

Apparaît ici une distinction entre le paîs et les néoi, pour lesquels le garçon est objet de souci. Ce qu'il convient de retenir de ces vers est, en premier lieu, le fait que le garçon est présenté comme un élément quasi inerte : sa beauté qui suscite le désir touche les amants par le biais du regard (le mot eîdos, "aspect», est lié au verbe eideîn, "voir»). On constate, deuxièmement, que les néoi sont accablés par le désir et qu'ils apparaissent comme les éléments actifs de la scène: ils cherchent à convaincre l'aimé d'instaurer une relation de philótēs (une réciprocité, impliquée par le mot cháris du vers 1321). Ici, la représentation du paîs est quasi identique à celle d'une jeune épouse dans un poème nuptial de Sappho, le fragment 112 : les deux, grâce à Aphrodite, se distinguent par leur beauté et le désir qu'ils suscitent chez ceux qui les regardent. Ce public, dans les deux cas, est semblable en terme de statut: pour le paîs, les néoi; pour la mariée, celui qui va bientôt quitter le statut de néos, le jeune homme en âge de se marier ${ }^{72}$.

La question de la persuasion est centrale dans ce contexte érotique. Dans les vers 1235-1238 des Theognidea, le poète veut persuader le paîs du fait qu'il ne devra pas faire ce qui est contraire à ces désirs. L'asymétrie, donc, ne signifie pas que le paîs reste inactif, livré aux

71 Théognis, v. 1319-1322 [W.2] (trad. Carrière modifiée).

72 Cf. supra Solon, fr. 27 [W.2]. 
désirs de l'amant: non seulement le paîs doit être persuadé d'accorder sa cháris, mais de plus il peut aussi avoir ses propres « appétits ».

Jeune garçon (paîs), tel un cheval, après t'être rassasié d'orge, tu es revenu sous mon toit, impatient d'y trouver bon cavalier, belle prairie, source fraîche et ombreux bocage. ${ }^{73}$

L'asymétrie érotique, on le voit, n'implique pas le fait que le paîs est un élément inactif. S’il est généralement dépeint comme étant la personne qui suscite le désir, qui s'enfuit et qui donne sa cháris alors que son amant doit le poursuivre, accablé par érōs et contraint à demander la réciprocité, l'aimé aussi peut subir le charme de celui qui l'aime et, suivant une image érotique traditionnelle, être comme un cheval cherchant son cavalier et le pâturage que celui-ci allait lui offrir ${ }^{74}$.

Quel statut social, alors, le paîs avait-il dans le banquet aristocratique? Il faut remarquer que les adolescents restaient assis sur des chaises ou que, dans certaines occasions, comme des échansons, ils servaient les adultes: cela est significatif, car les convives étaient généralement allongés sur des lits pendant le repas ${ }^{75}$. Les Theognidea offrent des éléments importants à ce propos: aux vers 27-38, instructifs pour l'éducation aristocratique de l'époque archaïque, le poète conseille au paîs de fréquenter les agathoí, les nobles, car il pourra apprendre des bonnes choses de la part des gens qui sont bons; le poète exhorte l'adolescent à boire et manger avec des hommes nobles et bons et, surtout, à s'asseoir parmi eux (v. 33 sq. metà toîsin híze $)^{76}$. Si, comme il est vraisemblable, le banquet couché avait déjà été introduit en Grèce à l'époque des Theognidea, le fait qu'un paîs restait assis pouvait marquer la différence de statut social entre adultes et adolescents.

Le cadre que l'on a esquissé pour l'érōs des hommes concerne d'une part le désir envers les parthénoi et d'autre part celui pour les paîdes: ce sont, sans doute, des rapports asymétriques. Dans ces relations, pourtant, la question de la persuasion est fondamentale.

\footnotetext{
73 Théognis v. 1249-1252 [W.2] (trad. Carrière modifiée).

74 Cf. Alcman, fr. 1, 47 sq. [D.] ; Sappho, fr. 2, 9 [V.] et, en outre, Neri 2011 : 264.

75 Cf. Bremmer 1990: 139 sq. et Caciagli 2011 : 93 et 168 sq. À propos du banquet couché, cf. Dentzer 1982.

76 Cf. aussi Alcée, fr. 75, 7 sq. [V.].
} 
Archiloque, poète parien du VII siècle, représente dans un poème ${ }^{77}$ la séduction d'une parthénos (v. 42), qui est la fille de son adversaire Lycambès et la sœur de Néoboulè, son ancienne fiancée. Le locuteur a comme objectif de convaincre la parthénos: il évoque la bonne mère de celle-ci (v. 7 sq.) ; pour lui montrer son respect, il lui explique que son but n'est pas la chose divine (v. 10 theîn chrêma, à savoir le rapport sexuel complet ${ }^{78}$, car d'autres plaisirs propres aux jeunes hommes (v. 9 néoisin andrásin) lui suffiront; il invite, surtout, la fille à se laisser persuader d'accomplir ce qu'il lui demande (v. 19). Là, on le voit, la parthénos est présentée comme ayant, elle aussi, ses propres désirs. On trouve une situation semblable dans un poème de Sappho ${ }^{79}$, où la locutrice avoue à sa mère qu'elle n'arrive plus à tisser, car elle est accablée par le désir qu'elle ressent pour un $p a \hat{s}{ }^{80}$.

\section{Une symétrie féminine?}

L'érōs entre femmes était-il différent de l'érōs entre hommes? Les risques de surinterprétation sont nombreux, en raison de projections anachroniques auxquelles l'homoérotisme féminin a longtemps donné lieu. Un exemple représentatif de ces lectures anachroniques est celui de la prétendue symétrie de l'érōs féminin, une symétrie qui s’opposerait à l'asymétrie de l'érōs masculin. Selon certaines interprétations, hors de la nécessité de domination typique des sociétés patriarcales, les femmes grecques auraient connu des rapports érotiques où la réciprocité était absolue et l'affection véritable ${ }^{81}$.

Cette lecture est trop rigide et il suffit d'analyser quelques documents pour l'infirmer. Alcman, poète spartiate du VII ${ }^{\mathrm{e}}$ siècle, compose, entre autres, des poèmes destinés à des chœurs de jeunes filles. Celles-ci chantent leur admiration et leur élan érotique pour plusieurs figures féminines. L'élan érotique, qui apparaît dans deux

\footnotetext{
77 Archiloque, fr. 196a [W.2].

78 Cf. Degani 1977 : 20-23 ; Degani 2005 [1977] : 13.

79 Cf. Sappho, fr. 102 [V.].

80 Bien que paîs vaille pour les filles et les garçons, ces vers semblent avoir un contexte nuptial, ce qui permet de penser que paîs est masculin.

81 Cf. Pippin Burnett 1983 : 226 ; Cantarella 1991 [1988] : 129 sq. ; cf. Dover 1982 [1978] : 209-224.
} 
contextes, se formule selon une situation exactement inverse à ce que l'on trouve dans les poèmes érotiques dits pédérastiques. Dans le fragment $1^{82}$, le locuteur correspond tour à tour à une parthénos (v. 86) et aux choreutes (v. 68 et v. 9983): la chorège, Hagésichora, est représentée sur un plan supérieur, en particulier en ce qui concerne sa beauté et sa capacité à chanter ${ }^{84}$. Rien n'est dit sur son âge, mais elle pourrait être la plus âgée des choreutes en raison de sa fonction ${ }^{85}$. L'éloge de sa beauté fait penser que le chœur éprouve de l'érōs pour elle, mais, si c'est le cas, l'élément qui est au niveau supérieur, la chorège, est l'objet du désir de celles qui sont inférieures à elle ${ }^{86}$.

Le fragment 3 d'Alcman présente une situation semblable: la locutrice, sûrement jeune, est à un niveau inférieur d'Astymeloisa, car elle se présente comme sa suppliante (v. 81) ; Astymeloisa, par contre, est «objet de sollicitude pour le peuple» (v. 74 mélèma dámōi $i^{87}$ ). Délicate (v. 68), elle est comparée à une pousse (v. 68) et elle possède la cháris (v. 71). Ce qui est intéressant est qu'Astymeloisa, objet du désir du «je» (v. 61-63), ne lui répond pas $^{88}$ : comme dans le fragment 1, la personne qui occupe une position supérieure est donc représentée comme l'objet du désir de celles qui lui sont inférieures. Cette situation problématique est peut-être la conséquence du fait que les hommes étaient membres de l'auditoire de ces deux poèmes : aux yeux des hommes, la jeune fille est toujours objet du désir. Le point de vue des locuteurs, alors, exprime également celui de l'auditoire.

Le cas des poèmes de Sappho est différent, car certains d'entre eux présupposent un auditoire complétement féminin ${ }^{89}$. L'existence d'une asymétrie dans l'érōs est essentielle pour comprendre les fragments 22

82 Pour une interprétation globale du fr. 1 [D.] d'Alcman, cf. Caciagli 2009.

83 Les commentaires antiques au fr. 1 [D.] d'Alcman indiquent que les choreutes sont des parthénoi.

84 Cf. v. $45-59$ et v. 90-101.

85 Cf. Calame 1977 : II, 98-104.

86 Les v. 70-76 du fr. 1 [D.] pourraient impliquer de l'érōs symétrique entre jeunes filles, si on les identifie avec les choreutes : cette identification, pourtant, n'est pas nécessaire.

87 Cf. supra Théognis, v. 1320 [W.2].

88 Cf. infra Anacréon, fr. 360 [P.].

89 Cf. Aloni 1997 : IX sq. 
ou 96, où Sappho n'est pas protagoniste du rapport érotique, mais simplement assiste à un lien entre deux femmes. Il est important d'utiliser ici le terme de "femmes», car l'idée que les «amies», les philai, de Sappho étaient toutes des jeunes filles peut être un préjugé contemporain ou une surinterprétation: dans le fragment 94, par exemple, l'interlocutrice de Sappho est souvent considérée par les commentateurs comme une parthénos en train de quitter Sappho pour se marier, mais cette supposition n'a aucun appui dans le texte. Quoi qu'il en soit, le fragment 1 (où la poétesse s'adresse à Aphrodite) met en scène une configuration érotique et un discours similaire à ceux que nous trouvons dans les relations homoérotiques masculines chantées par les poètes archaïques. Dans le poème de Sappho, en effet, la personne qui aime est accablée par le désir (v. 3 sq.) et la personne aimée ne répond pas au sentiment que l'on éprouve pour elle (v. 18) : l'érōs est un piège, un dólos (v. 2); le manque de réciprocité est une injustice, une adikía (v. 20) ${ }^{90}$; la persuasion est un élément nécessaire (v. 15) ; la relation érotique, enfin, implique la fuite et la poursuite, avec l'échange de dons (v. 21-22).

En effet, si elle s'enfuit, bientôt elle poursuivra, si elle n'accepte pas de dons, elle en donnera pourtant, si elle n'aime pas, bientôt elle aimera

même contre sa volonté.

Cette strophe a probablement une valeur générale, car les verbes des vers 21 et 23 n'ont pas de complément d'objet : celle qui parle, Aphrodite, assure que l'aimée ${ }^{91}$ non seulement cessera de fuir et acceptera les dons qu'on lui offre, mais qu'elle poursuivra et fera des dons à son tour. Deux possibilités s'ouvrent : soit il s'agit de dire que l'aimée se trouvera un jour dans la même situation que la personne qui aime ${ }^{92}$ et qu'il s'agit ici de l'annonce d'une sorte de vengeance pour cette dernière ${ }^{93}$; soit, la déesse assure, dans le futur, la réciprocité totale entre la personne qui aime et la personne qui est

\footnotetext{
90 Cf. Théognis, v. 1282 sq. [W.2].

91 Pour le genre de l'aimée, cf. le participe féminin ethéloisa (« voulant ») du v. 22.

92 Cf. Théognis, v. 1329-1334 [W.2].

93 Cf. Giacomelli 1980.
} 
l'objet de cette philótès $^{94}$. La première option, cependant, n'est pas vraisemblable, car elle n'entre pas en cohérence avec la requête que Sappho fait à Aphrodite : achever «ce que son cœur désire qu'il soit achevé » (v. 26 sq. $)^{95}$, à savoir que son aimée veuille établir avec elle une relation de philótés 96 .

L'interprétation qui va dans le sens d'une réciprocité totale n'implique cependant pas la symétrie: la philótès est un type de relation qui suppose une réciprocité souvent asymétrique. Si un paîs masculin ou féminin peut non seulement recevoir les dons de l'amant mais aussi donner sa cháris, si un paîs peut chercher son amant et le désirer ${ }^{97}$, un rapprochement entre la philótès érotique et le rapport de compagnonnage, l'hetaireía, pourrait être significatif: l'hetaireía, en effet, n'est rien d'autre qu'une déclinaison de la relation de philótès ${ }^{98}$. Or, un des rapports considéré comme paradigmatique du compagnonnage guerrier, celui entre Achille et Patrocle, montre que la réciprocité entre les deux partenaires ne se base pas sur une égalité d'âge ou de statut social : Patrocle, qui est le serviteur d'Achille ${ }^{99}$ et a même peur de lui ${ }^{100}$, est plus âgé qu'Achille ${ }^{101}$, mais inférieur en ce qui concerne la force. La condition de supériorité, pourtant, n'empêche pas Achille de faire cesser sa colère pour venger la mort de son compagnon Patrocle, lui qui émet le souhait d'un tombeau commun avec son phílos hetaîros ${ }^{102}$.

Les notions d'asymétrie et de symétrie dans les relations érotiques sont très souvent utilisées dans les analyses des spécialistes de l'Antiquité pour lire les documents évoquant la sexualité antique, mais leurs analyses se heurtent à des projections anachroniques portant sur les identités sexuelles. Ce qui apparait de l'analyse des documents est que, en Grèce ancienne, érōs semble instaurer une relation

\footnotetext{
94 Cf. Dover 1982 [1978] : 217-218.

95 Sappho, fr. 1, v. $26 s q[\mathrm{~V}$.$] .$

96 Cf. Sappho, fr. 1, v. 18-20 [V.].

97 Cf. Théognis, v. 1249-1252.

98 Cf. Caciagli 2011 : 56 sq.

99 Cf. Iliade, XVIIII, 151 sq.

100 Cf. Iliade, XI, 649-654.

101 Cf. Iliade, XI, 786-789.

102 Cf. Iliade, XXIII, 77-83.
} 
asymétrique, et ce quel que soit le sexe des amants : le désir engendre une course, une fuite, des refus, de l'attente, et les relations de domination s'inversent fréquemment dans les tableaux que donnent à entendre les vers des poètes archaïques, qu'il s'agisse de poétesse (Sappho) ou de poètes (Alcman, les Theognidea, Anacréon). La réciprocité est ce à quoi aspire érōs et, dans cette configuration, asymétrie et réciprocité ne sont pas contradictoires.

Certaines lectures, ainsi, ont souvent rendu trop figées une réalité, celle des pratiques grecques archaïques liées à érōs et à la sexualité des pratiques qui étaient bien plus variées et complexes que les concepts opératoires modernes de relation sociale de sexe ou que les équivalences entre "pénétration sexuelle» et hiérarchisation sociale. Les notions de symétrie ou d'asymétrie, d'égalité ou de domination, parlent moins des Grecs que des savants qui les étudient. À la question «Que met en jeu érōs? ?, un ou une Grec(que) de l'époque archaïque aurait probablement répondu, tout simplement: «La réciprocité (philótés)».

\section{Sources antiques}

Les textes originaux des auteurs antiques cités dans cet article peuvent être consultés dans les éditions de la CUF (Collection des Universités de France), publiées aux éditions des Belles Lettres, à l'exception des auteurs suivants :

Alcée [V.] = Voigt, Eva-Maria, 1971, Sappho et Alcaeus. Fragmenta, Amsterdam, Polak \& van Gennep.

Alcman [D.] = Davies, Malcom, 1991, Poetarum melicorum graecorum fragmenta, Oxford, Oxford University Press.

Anacréon [P.] = Page, Denis L., 1962, Poetae melici graeci, Oxford, Oxford University press.

Apollonius Dyscole, Syntaxe = Lallot, Jean, 1997, Apollonius Dyscole, De la construction, Paris, Vrin.

Archiloque [W.2] = West, Martin L., 1989-1992, Iambi et elegi Graeci ante Alexandrum cantati. Editio altera, I-II, Oxford, Oxford University

Code de Gortyne, IC = Halbherr, Federico \& Guarducci, Margherita, 1935-1950, Inscriptiones Creticae, I-IV, Roma, Libreria dello Stato. 
Diphile $=$ Kassel, Rudolf \& Austin, Colin, 1983-2001, Poetae comici Graeci, I-VIII, Berlin, W. de Gruyter.

Antiphane = Kassel, Rudolf \& Austin, Colin, 1983-2001, Poetae comici Graeci, I-VIII, Berlin, W. de Gruyter.

Eubule $=$ Kassel, Rudolf \& Austin, Colin, 1983-2001, Poetae comici Graeci, I-VIII, Berlin, W. de Gruyter.

Eustathe, Commentaire à l'Odyssée = Stallbaum, Johann Gottfried, 1825-1826, Eustathii archiepiscopi Thessalonicensis commentarii ad Homeri Odysseam, I-II, Leipzig, Weigel (reprint Hildesheim, Olms, 1970).

Mimnerme [W.2] = West, Martin L., 1989-1992, Iambi et elegi Graeci ante Alexandrum cantati. Editio altera, I-II, Oxford, Oxford University press.

P. Oxy. 1800, fr. 1 = Grenfell, Bernard P. \& Hunt, Arthur S., The Oxyrhynchus Papyri, $\mathrm{XV}$, London, Egypt exploration fund.

Pindare, Olympique = Maehler, Herwig, 1987-1989, Pindarus. Epinicia, fragmenta, indices, III, Leipzig, W. de Gruyter.

Pindare, Pythiques = Maehler, Herwig, 1987-1989, Pindarus. Epinicia, fragmenta, indices, I-II, Leipzig, W. de Gruyter.

Sappho [V.] = Voigt, Eva-Maria, 1971, Sappho et Alcaeus. Fragmenta, Amsterdam, Polak \& van Gennep.

Lycophron, scholies $\grave{a}=$ E. Scheer, Lycophronis Alexandra, vol. 2, Berlin: Weidmann, 1958

Solon [W.2] = West, Martin L., 1989-1992, Iambi et elegi Graeci ante Alexandrum cantati. Editio altera, I-II, Oxford, Oxford University press.

Éphore (FGrHist 70 F 149) = Jacoby, Felix, 1923-1999, Die Fragmente der griechischen Historiker, I-IV, Berlin, Weidmann.

Théognis [W.2] = West, Martin L., 1989-1992, Iambi et elegi Graeci ante Alexandrum cantati. Editio altera, I-II, Oxford, Oxford University press.

\section{Bibliographie}

Aloni Antonio, 1997, Saffo. Frammenti, Firenze, Giunti.

BENVENISTE Émile, 1969, Le Vocabulaire des institutions indo-européennes, Paris, Éditions de Minuit.

Blondell Ruby \& Kirk Ormand (eds), 2015, Ancient Sex: new essays, Columbus, $\mathrm{OH}$, Ohio State University Press.

BoeHringer Sandra, 2007a, L'Homosexualité féminine dans l'Antiquité grecque et romaine, Paris, Les Belles lettres. 
—, 2007b, «Comparer l'incomparable. La sunkrisis érotique et les catégories sexuelles dans le monde gréco-romain ", in Bruno PERreAu (éd.), Le Choix de l'homosexualité. Recherches inédites sur la question gay et lesbienne, Paris, Epel, p. 39-56.

—, 2012, «Le genre et la sexualité. États des lieux et perspectives dans le champ des études anciennes », Lalies, 32, p. 145-167.

—, 2014, «Existe-t-il une "homosexualité initiatique" ? Pour une anthropologie de la sexualité antique », in Alice Mouton \& Julie Patrier (eds), Life, Coming of Age and Death in Antiquity: individual rites of passage in the Ancient Near East, Leiden, The Nederlands Institute for the Near Est, p. 481-506.

Boehringer Sandra \& Antoine CHABOD, à paraitre 2015, «Chanter Tithon et Atalante (Sappho, Théognis) » in Irini Tsamadou-Jacoberger \& Marie BizAIS (éd.), Éducations sentimentales. La Construction des identités féminines et masculines dans le texte et l'image, Strasbourg, Presses universitaires de Strasbourg.

Boehringer Sandra \& Louis-Georges Tin (éd.), 2010, Homosexualité. Aimer en Grèce et à Rome, Paris, Les Belles Lettres.

Brelich Angelo, 1969, Paides e Parthenoi, Rome, Ed. Dell'Ateneo, coll. «Incunabula graeca », 36.

Bremmer Jan N., 1990, "Adolescents, symposion, and pederasty », in Oswyn Murray (ed.), Sympotica: a symposium on the symposion, Oxford, Oxford University Press, p. 135-148.

BRISSON Luc, 2000, « Le Banquet de Platon comme document sur les comportements sexuels et leur représentation sociale », in Louis-Georges TIN (dir.), Homosexualités. Expression/répression, Paris, Stock, p. 49-62.

—, 2006a, «Agathon, Pausanias, and Diotima in Plato's Symposium: Paiderastia and Philosophia? ", in James H. Lesher, Debra NAils \& Frisbee SHEFFIELD (eds), Plato's Symposium. Issues in Interpretation and Reception. Cambridge, MA, Harvard University Press, coll. «Hellenic Studies », 22, p. 229-251.

—, 2006b, «Préface » de Platon, Les Lois (Livres I à VI et Livres VII à XII), Paris, Flammarion.

—, 2015, «Éros éducateur: entre paiderastía et philósophia », in Mauro Tulli (éd.), Plato's Symposium, Symposium Platonicum X, Sankt Augustin, Academia Verlag (à paraitre).

Brulé Pierre, 1987, La Fille d'Athènes, Paris, Les Belles Lettres.

Caciagli Stefano, 2009, «Un contesto per Alcm. PMGF 1 », Eikasmós, 20, p. 19-46.

—, 2011, Poeti e società. Comunicazione poetica e formazioni sociali nella Lesbo del 7.-6. secolo a.C., Amsterdam, Adolf M. Hakkert.

CALAME Claude, 1977, Les Chxurs de jeunes filles en Grèce archä̈que, I : Morphologie, fonction religieuse et sociale, II : Alcman, Rome, Edizioni dell'Ateneo e Bizzari, coll. «Filologia e critica », 20 et 21 . 
—, (éd.), 1983, Alcman. Introduction, texte critique, témoignages, traduction et commentaire, Rome, in aedibus Athenaei, coll. « Lyricorum graecorum quae exstant », 6.

—, 1986, Le Récit en Grèce ancienne. Énonciation et représentation des poètes, Paris, Belin.

—, 1996, L'Éros dans la Grèce antique, Paris, Belin [trad. de : CALAmE Claude, I Greci e l'eros, par Maria Rosaria Falivene, Rome-Bari, Laterza, 1992].

—, 2002, «Interprétation et traduction des cultures. Les catégories de la pensée et du discours anthropologiques », L'Homme, CLXIII, p. 51-78.

_, 2003, «Le rite d'initiation tribale comme catégorie anthropologique (Van Gennep et Platon) », Revue de l'histoire des religions, CCXX/1, p. 5-62.

—, 2005, Masques d'autorité : fiction et pragmatique dans la poétique grecque antique, Paris, Les Belles Lettres.

Calame Claude, Dupont Florence, Lortat-Jacob Bernard \& Maria Manca (éd.), 2010, La Voix actée. Pour une nouvelle ethnopoétique, Paris, Anthropologie.

Cantarella Eva, 1991 [1988], Selon la nature, l'usage et la loi. La Bisexualité dans le monde antique, Paris, La Découverte [trad. fr. par Marie-Domitille Porcheron de Secondo natura, Milano, Rizzoli, 1988 (2e éd. 1995)].

Cantarella Eva \& Andrew Lear, 2008, Images of ancient Greek Pederasty: boys were their gods, London \& New York, Routledge.

Carrière Jean 1975, Théognis. Poèmes élégiaques, Paris, Les Belles Lettres.

Chantraine Pierre, 1968-1980, Dictionnaire étymologique de la langue grecque, Paris, Éditions Klincksieck.

CoHen David, 1991, "Sexuality, violence, and the Athenian law of Hubris», Greece and Rome, XXXVIII, p. 171-188.

COHEN Edward E., 2006, «Free and unfree sexual work: an economic analysis of Athenian prostitution », in Christopher A. FARAONE \& Laura A. MCCluRE (eds), Prostitutes and Courtesans in the Ancient World, Madison, University of Wisconsin Press, p. 95-124.

—, 2014, "Sexual abuse and sexual rights: slaves' erotic experience at Athens and Rome », in Thomas K. HubBard, A Companion to Greek and Roman Sexualities, Malden, MA, Wiley Blackwell, p. 184-198.

Colesanti Giulio, 2011, Questioni teognidee, Roma, Edizioni di storia e letteratura.

Davidson Arnold, 2005, L'Émergence de la sexualité, Paris, Albin Michel [trad. fr. par Pierre-Emmanuel Dauzat de The Emergence of Sexuality: historical epistemology and the formation of concepts, Cambridge, MA, Harvard University Press, 2001].

DAvies Malcom, 1991, Poetarum melicorum Graecorum fragmenta, Oxford, Oxford University Press. 
Degani Enzo, 1977, «Sul nuovo Archiloco », in Degani Enzo (éd.), Poeti greci giambici ed elegiaci, Milano, Mursia, p. 15-43.

Degani Enzo \& Gabriele Burzacchini, 2005 [1 1re éd. 1977], Lirici greci. Antologia, Bologna, Patron.

Dentzer Jean-Marie, 1982, Le Motif du banquet couché dans le Proche-Orient et le monde grec du VII au IVe siècle avant J.C., Roma, École française de Rome.

Dover Kenneth J., 1982, Homosexualité grecque, Grenoble, La pensée sauvage, coll. «Bibliothèque d'ethnopsychiatrie» 4 [trad. fr. par Suzanne Saïd de Greek Homosexuality, London, Duckworth, 1978].

DUPONT Florence, 2010, «Introduction », in Florence DupOnT, Bernard LORTATJACOB \& Maria MANCA (éd.), La Voix actée. Pour une nouvelle ethnopoétique, Paris, Kimé, p. 7-20.

FERRARESE Estelle, 2009, «Vivre à la merci. Les trois figures de la vulnérabilité dans les théories politiques contemporaines », Multitudes, 37-38, p. 132-142.

—, 2011, "Les vulnérables et le géomètre. Sur les usages du concept de vulnérabilité dans les sciences sociales », Raison Publique, XIV, p. 17-37.

Foucault Michel, 1984, Histoire de la sexualité, t. III : Le Souci de soi, Paris, Bibliothèque des histoires.

GARLAN Yvon, 1982, Les Esclaves en Grèce ancienne, Paris, La Découverte.

Gentili Bruno, 2006 [1 1 éd. 1984], Poesia e pubblico nella Grecia antica, Milano, Feltrinelli editore.

Giacomelli Anne, 1980, «The justice of Aphrodite in Sappho fr. 1 », Transaction of American Philological Association, CX, p. 135-142.

Gilhully Kate, 2015, «Lesbians are not from lesbos », in Ruby BLONDELL \& Kirk Ormand (eds), Ancient Sex: new essays, Columbus, OH, Ohio State University Press, p. 143-176.

GlazeBrooK Allison \& Kelly OLSON, 2014, "Greek and roman marriage », in Thomas K. Hubbard (ed.), A Companion to Greek and Roman Sexualities, Malden, Ma, Wiley Blackwell, p. 69-82.

HALPERIN David, 1992, "Historicizing the subject of desire: sexual preferences and erotic identities in the Pseudo-Lucianic Erotes)», in Domna C. STANTON (ed.), Discourses of Sexuality: from Aristotles to AIDS, Ann Arbor, University of Michigan Press, p. 236-261 [réédité in Jan GoldsTEIN (ed.), Foucault and the Writing of History, Oxford, Blackwell, 1994, p. 19-34 et p. 255-261].

—, 2000, Cent ans d'homosexualité et autres essais sur l'amour grec, Paris, Epel [trad. fr. par Isabelle Châtelet de One Hundred Years of Homosexuality, New York \& London, Routledge, 1990]. 
Halperin David M., WinkLer John J. \& Froma I. Zeitlin (eds), 1990, Before Sexuality: the construction of erotic experience in the Ancient World, Princeton, Princeton University Press.

HubBard Thomas K., 2003, Homosexuality in Greece and Rome: a sourcebook of basic documents, Berkeley, University of California Press.

-, (éd.), 2014a, A Companion to Greek and Roman Sexualities, Malden, Ma, Wiley Blackwell.

—, 2014b, «Peer homosexuality», in Thomas K. HubBard (ed.), A Companion to Greek and Roman Sexualities, Malden, Ma, Wiley Blackwell, p. 128-149.

JeAnMaire Henri, 1939, Couroi et Courètes. Essai sur l'éducation spartiate et sur les rites d'adolescence dans l'Antiquité hellénique, Lille, Bibliothèque universitaire de Lille.

KATz Jonathan N., 2001. L'Invention de l'bétérosexualité, Paris, Epel [trad. fr. par Michel Oliva, Éliane Sokol et Catherine Thévenet de The Invention of Heterosexuality, New York, Dutton Books, 1995].

KEnNEL Nigel M., 1995, The Gymnasium of Virtue: education \& culture in ancient Sparta, Chapel Hill, NC \& London, University of North Carolina Press.

LEAR Andrew, 2014, "Ancient pederasty: an introduction », in Thomas K. HubBard (ed.), A Companion to Greek and Roman Sexualities, Malden (Mass.), Wiley Blackwell, p. 102-127.

LEDuC Claudine, 1991, "Comment la donner en mariage ? La mariée en pays grec », in Pauline Schmitt Pantel (dir.), L'Antiquité, volume I de Georges Duby \& Michelle Perrot (dir.), L'Histoire des femmes en Occident, Paris, Plon, p. 259-280.

Murray Oswyn (ed.), 1990, Sympotica: a Symposium on the Symposion, Oxford, Oxford University Press.

Neri Camillo, 2011, Lirici greci: età arcaica e classica, Roma, Carocci.

OAKLey John H. \& Rebecca H. Sinos, 1994, The Wedding in Ancient Athens, Madison, WI, University of Wisconsin Press.

ORMAND Kirk, 2014, "Foucault's History of Sexuality and the discipline of classics ", in Thomas K. HubBard (ed.), Homosexuality in Greece and Rome: a sourcebook of basic documents, Berkeley, University of California Press, p. 54-68.

— 2016, «Peut-on parler de perversion dans l'Antiquité ? Foucault et l'invention du raisonnement psychiatrique », trad. Sandra Boehringer \& Isabelle Châtelet, in Sandra Boehringer \& Daniele Lorenzini, Foucault, l'Antiquité, la sexualité, Paris, Kimé.

Page Denys L., 1962, Poetae melici Graeci, Oxford, Oxford University Press.

Perusino Franca, 2002, «Le orse di Brauron nella Lysistrata di Aristofane », in Bruno Gentili \& Franca Perusino (a cura di), Le Orse di Brauron: un rituale di iniziazione femminile nel santuario di Artemide, Pisa, ed. ETS, p. 167-174. 
Pippin Burnett Anne, 1983, Three Archaic Poets. Archilochus, Alcaeus, Sappho, London, Duckworth.

ReNAuT Olivier, 2015, «La pédérastie selon Pausanias: un défi pour l'éducation platonicienne ? ", in Mauro Tuldi (ed.), Plato's Symposium, Symposium Platonicum X, Sankt Augustin, Academia Verlag (à paraître).

—, 2016, "Genre et sexualité dans les Républiques de Platon, Diogène et Zénon », in Juliette Lemaire \& Suzanne Husson (éd.), Les Trois Républiques : Platon, Diogène, Zénon, Paris, J. Vrin (à paraître).

RÖSLER Wolfgang, 1980, Dichter und Gruppe, München, W. Fink.

-, 1984, Die frühe griechische Lyrik und ibre Interpretation. Versuch einer Situationsbeschreibung, Poetica, 20, p. 179-205.

SCHMitT-PANTEL, Pauline, 1992, La Cité au banquet : histoire des repas publics dans les cités grecques, Rome, Publications de l'École française de Rome.

SERGENT Bernard, 1996 [1 re éd. 1984], L'Homosexualité dans la mythologie grecque, Paris Payot et 1996 [1 1re éd. 1986], L'Homosexualité initiatique dans l'Europe ancienne, Paris, Payot, réédités conjointement en 1996 : Homosexualité et initiation chez les peuples indo-européens, Paris, Payot.

Stansbury-O’Donnell Mark D., 2014, «Desirability and the Body », in Thomas K. Hubbard (ed.), Homosexuality in Greece and Rome: a sourcebook of basic documents, Berkeley, University of California Press, p. 31-53.

VAN GRONINGEN Bernhard Abraham, 1966, Théognis. Le premier livre édité avec un commentaire, Amsterdam, Noord-Hollandsche Uitgevers Maatschappij.

VetTA Massimo, 1980, Teognide. Elegie, libro II, Roma, Edizioni dell'Ateneo.

—, 1983, Poesia e simposio nella Grecia antica: guida storica e critica, Roma, Laterza.

Voigt Eva-Maria, 1971, Sappho et Alcaeus. Fragmenta, Amsterdam, Athenaeum-Polak \& Van Gennep.

WEST Martin Litchfield, 1974, Studies in Greek Elegy and Iambus, Berlin and New York, Walter de Gruyter.

—, $1989^{2}$ (vol. I) et $1992^{2}$ (vol. II), Iambi et elegi Graeci, Oxford, Oxford University Press.

Winkler John J., 2005, Désir et contraintes en Grèce ancienne, Paris, Epel [trad. fr. par Sandra Boehringer et Nadine Picard de The Constraints of Desire. The Anthropology of Sex and Gender in Ancient Greece, New York \& London, Routledge, 1990]. 